\title{
ON MINIMIZING NONCOERCIVE FUNCTIONALS ON WEAKLY CLOSED SETS
}

\author{
VY KHOI LE and KLAUS SCHMITT \\ Department of Mathematics \\ University of Utah \\ Salt Lake City, UT 84112, USA \\ E-mail:schmitt@math.utah.edu
}

\begin{abstract}
We consider noncoercive functionals on a reflexive Banach space and establish minimization theorems for such functionals on smooth constraint manifolds. The functionals considered belong to a class which includes semi-coercive, compact-coercive and P-coercive functionals. Some applications to nonlinear partial differential equations are given.
\end{abstract}

1. Introduction. Let $V$ be a real reflexive Banach space with dual $V^{*}$ and the pairing between $V$ and $V^{*}$ denoted by $\langle\cdot, \cdot\rangle$ and the norm by $\|\cdot\|$. Let

$$
F: V \rightarrow \mathbb{R} \cup\{\infty\}
$$

be a weakly lower semicontinuous functional and let $S \subset V$ be a weakly closed set. If it is the case that $F$ is coercive, then it is a classical result that there exists $u \in S$ such that

$$
F(u)=\min _{v \in S} F(v) .
$$

(See e.g.[MW],[St].) On the other hand, if $F$ is not necessarily coercive but there exists a weakly lower semicontinuous nonnegative functional

$$
G: V \rightarrow \mathbb{R}_{+} \cup\{\infty\}
$$

such that the perturbed functionals

$$
F_{\epsilon}(u)=F(u)+\epsilon G(u)
$$

1991 Mathematics Subject Classification: Primary 35J15; Secondary 35J85, 49J40.

Key words and phrases: minimization of functionals, variational inequalities, semi-coercive functional, P-coercive functional.

Research supported by a grant from NSF.

The paper is in final form and no version of it will be published elsewhere. 
are coercive for $\epsilon>0$ and that the solution sets $\left\{u_{\epsilon}\right\}$, of

$$
F_{\epsilon}(u)=\min _{v \in S} F_{\epsilon}(v)
$$

are a priori bounded, then a solutions of the original problem exists and may be obtained via a limit process, letting $\epsilon \rightarrow 0$. For, since $V$ is reflexive, the set $\left\{u_{\epsilon}\right\}$, being assumed bounded, will have a weakly convergent subsequence, say $\left\{u_{\epsilon_{n}}\right\}, u_{\epsilon_{n}} \rightarrow u$, where $\rightarrow$ denotes weak convergence. Hence, by the weak lower semicontinuity

$$
\begin{aligned}
F(u) & \leq \liminf _{n \rightarrow \infty} F\left(u_{\epsilon_{n}}\right) \\
& \leq \liminf _{n \rightarrow \infty} F_{\epsilon_{n}}\left(u_{\epsilon_{n}}\right) \\
& \leq \liminf _{n \rightarrow \infty} F_{\epsilon_{n}}(v), v \in S \\
& =F(v), v \in S .
\end{aligned}
$$

This regularization procedure has been used extensively and dates back (for functionals of a special from) to at least [F] and [LS] and has been widely used (see e.g.[KS], [ASV1$3],[\mathrm{AV}],[\mathrm{BG}],[\mathrm{BGT}],[\mathrm{H}],[\mathrm{LS} 1-2],[\mathrm{S}])$. The procedure, of course will work, whenever the above conditions hold, i.e. we can find a regularizer $G$ and we can show that the solution set of (1.2) is a priori bounded.

We here provide a class of functionals (functionals with property $(P)$ ) (and compatible manifolds $S$ ), a framework, general enough, to include most of the above cited methods of attack, and include some applications to boundary value problems for nonlinear elliptic partial differential equations.

\section{Functionals with property $(P)$}

2.1. Assumptions and notations. Let

$$
A: V \rightarrow V^{*}
$$

be a mapping such that the functional

$$
\varphi: V \rightarrow \mathbb{R}
$$

given by

$$
u \mapsto\langle A u, u\rangle
$$

is weakly lower semicontinuous. Further assume that

$$
j: V \rightarrow \mathbb{R} \cup\{\infty\}(\not \equiv \infty)
$$

is a convex lower semicontinuous (hence weakly lower semicontinuous) functional satisfying (without loss in generality) $j(0)=0$.

We shall assume $F$ is of the form

$$
F=\varphi+j
$$

Let $Y$ be a Banach space with norm $\|\cdot\|_{Y}$ and suppose that

$$
\psi: V \rightarrow Y
$$

is a completely continuous mapping. Let $\gamma \in Y$ be fixed and let

$$
S=\{u \in V: \psi(u)=\gamma\} \text {. }
$$


Since $\psi$ is completely continuous, i.e. continuous from $V$ endowed with the weak topology to $Y$ with the norm topology, $S$ is weakly closed in $V$. We shall also consider the case that $S$ is a closed convex set. We assume that $S \neq \emptyset$, that $j(u)<+\infty$, for some $u \in S$ and $\varphi$ is bounded from below on $S$ in the sense that there exist constants $c>0$ and $0 \leq \beta<2$ such that

$$
\varphi(u) \geq-c\|u\|^{\beta} \text {. }
$$

and we consider the minimization problem

$$
F(u)=\min _{v \in S} F(v) .
$$

Remark 1. If $S$ is a closed convex set, by changing the definition of $F$ to

$$
F=\varphi+j+I_{S}
$$

where

$$
I_{S}(u)=\left\{\begin{array}{l}
\infty, u \notin S \\
0, u \in S
\end{array}\right.
$$

is the indicator function of $S$, we obtain that problem $(2.3)$ is equivalent to

$$
F(u)=\min _{v \in V} F(v),
$$

with the modified $F$.

As before, we introduce a property which together with certain compatibility conditions on $S$ will imply the boundedness of the solution set of the regularized problems. As we shall see this property will be an extension of the concepts compact coerciveness and P-coerciveness used in the literature, e.g.[BGT], [BT], [ASV1-3], [AV].

\subsection{Property $(P)$.}

Definition. We say that the functional $F$ (or the pair $(\varphi, j)$ or $\varphi$, if $j=0$ ) has property $(P)$ on $S$ whenever the following hold: There exists a constant $p>1$ such that: If $\left\{v_{n}\right\} \subset S$ is any sequence in $S$ satisfying, as $n \rightarrow \infty$,

$$
\begin{cases}(a) & \left\|v_{n}\right\| \rightarrow \infty, \\ (b) & w_{n}=\frac{v_{n}}{\left\|v_{n}\right\|} \rightarrow w, \\ (c) \quad\left\|v_{n}\right\| \leq\left\|v_{n}-\lambda w\right\|, \forall n, \forall \lambda \geq 1, & \\ (d) \quad & \limsup _{n \rightarrow \infty} \frac{\varphi\left(v_{n}\right)}{\left\|v_{n}\right\|^{p}} \leq 0,\end{cases}
$$

then there exists $v_{0} \in S$ such that

$$
\limsup _{n \rightarrow \infty} F\left(v_{n}\right)>F\left(v_{0}\right) .
$$

(For a Banach space geometric interpretation of condition $(2.4$ (c)) see e.g. [J].)

We shall next give a sequence of sufficient conditions guaranteeing that property $(P)$ holds. 
Proposition 2.1. Suppose that $F$ satisfies the conditions of the definition with $(c)$ replaced by

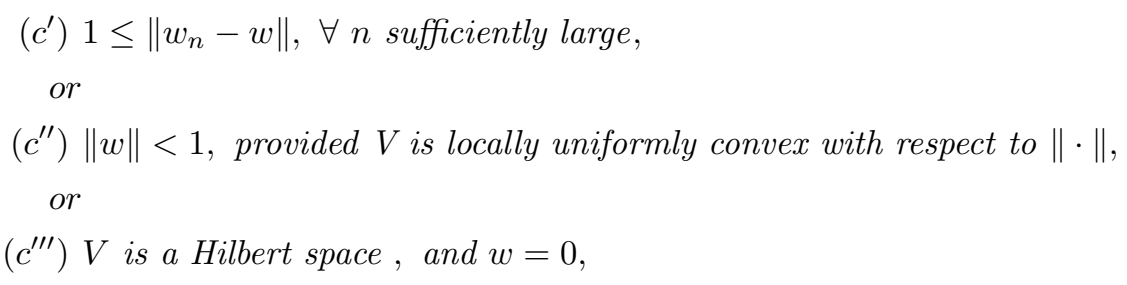

then $F$ satisfies property $(P)$.

Proof. Suppose $\left\{v_{n}\right\}$ and $F$ are as above. We label the cases to be considered as $\left(c^{\prime}\right),\left(c^{\prime \prime}\right),\left(c^{\prime \prime \prime}\right)$.

Case $\left(c^{\prime}\right)$ : Since (by (a)) $\left\|v_{n}\right\| \geq 1$, for $n$ sufficiently large, we have from (c) that

$$
\left\|v_{n}\right\| \leq\left\|v_{n}-\right\| v_{n}\|w\|, \forall n \text { sufficiently large, }
$$

hence

$$
1 \leq\left\|w_{n}-w\right\|, \forall n \text { sufficiently large, }
$$

and property $(P)$ holds.

Case $\left(c^{\prime \prime}\right)$ : We first note that if $V$ is reflexive then we may choose (by the Lindenstrauss - Asplund - Trojanski theorem [PS] or [T]) an equivalent norm on $V$ such that $V$ is locally uniformly convex with respect to the new norm. The local uniform convexity of $V$ yields the property that (see e.g.[B])

$$
u_{n} \rightarrow u, \text { whenever } u_{n} \rightarrow u \text { and }\left\|u_{n}\right\| \rightarrow\|u\| .
$$

Suppose then that the conditions of the definition hold for a sequence $\left\{v_{n}\right\}$ but that $\left(c^{\prime \prime}\right)$ is false, i.e. $\|w\| \geq 1$. We then have

$$
\limsup _{n \rightarrow \infty}\left\|w_{n}\right\|=1 \leq\|w\|
$$

however, since $\|\cdot\|$ is lower semicontinuous, it follows that

$$
\|w\| \leq \liminf _{n \rightarrow \infty}\left\|w_{n}\right\|
$$

and hence

and thus by the above property

$$
\|w\|=\lim _{n \rightarrow \infty}\left\|w_{n}\right\|
$$

$$
w_{n} \rightarrow w,
$$

contradicting the fact that for $n$ large

$$
1 \leq\left\|w_{n}-w\right\|
$$

which follows from (c).

Case $\left(c^{\prime \prime \prime}\right)$ : Suppose $\left\{v_{n}\right\}$ is a sequence as in the definition. Choose $\lambda=1$ in (c) and get

$$
\left\|v_{n}\right\|^{2} \leq\left\|v_{n}-w\right\|^{2}=\left\|v_{n}\right\|^{2}+\|w\|^{2}-2\left\langle v_{n}, w\right\rangle
$$


$(\langle\cdot, \cdot\rangle$ is the inner product of $V$ now $)$ and thus

implying that

$$
\left\langle w_{n}, w\right\rangle \leq \frac{\|w\|^{2}}{2\left\|v_{n}\right\|^{2}},
$$

hence $w=0$.

$$
\|w\|^{2}=\lim _{n \rightarrow \infty}\left\langle w_{n}, w\right\rangle=0
$$

Thus in either of the three cases we have shown that property $(P)$ holds.

Proposition 2.2. Assume that $F$ satisfies: If $\left\{v_{n}\right\}$ is a sequence in $S$ such that

$$
\begin{cases}\bullet & \left\|v_{n}\right\| \rightarrow \infty, \quad w_{n}=\frac{v_{n}}{\left\|v_{n}\right\|} \rightarrow w \\ \bullet & \sup _{n \in \mathbf{N}} F\left(v_{n}\right)<+\infty,\end{cases}
$$

then

Then $F$ has property $(P)$.

$$
w_{n}=\frac{v_{n}}{\left\|v_{n}\right\|} \rightarrow w
$$

Pro of. Let $\left\{v_{n}\right\} \subset S$ be a sequence such that (2.4) holds, we claim that

$$
\limsup _{n \rightarrow \infty} F\left(u_{n}\right)=+\infty
$$

and hence taking $v \in S$ such that $j(v)<\infty$ we complete the proof. If the claim were false we get that

$$
F\left(v_{n}\right) \leq c, \quad n \in \mathbf{N}
$$

for some constant $c$ and hence by assumption

$$
w_{n} \rightarrow 0,
$$

a contradiction to $\left\|w_{n}\right\|=1$.

The following example shows that the conditions given by proposition 2.2 are a strict special case of property $(P)$, even in the case of Hilbert spaces.

Example. Let $V$ be an infinite dimensional Hilbert space and let

$$
F(u)=\frac{\|u\|}{1+\|u\|} .
$$

We shall show that $F$ is weakly lower semicontinuous, has property $(P)$, yet does not satisfy the conditions of proposition 2.2. In fact, suppose that $\left\{u_{n}\right\}$ is a sequence with $u_{n} \rightarrow u$. Select a subsequence $\left\{u_{n_{k}}\right\} \subset\left\{u_{n}\right\}$ such that

$$
\lim _{k \rightarrow \infty} F\left(u_{n_{k}}\right)=\liminf _{n \rightarrow \infty} F\left(u_{n}\right) .
$$

Since $\left\{u_{n_{k}}\right\}$ is bounded, we may assume, by passing to a subsequence, if necessary, that $\left\{\left\|u_{n_{k}}\right\|\right\}$ is convergent. Moreover, by the weak lower semicontinuity of the norm, we have

$$
\|u\| \leq \lim _{k \rightarrow \infty}\left\|u_{n_{k}}\right\| .
$$

Since the mapping

$$
G: \mathbb{R}_{+} \rightarrow \mathbb{R}_{+}, \quad x \mapsto \frac{x}{1+x}
$$


is continuous and increasing, we get

$$
\begin{aligned}
F(u) & =G(\|u\|) \leq G\left(\lim _{k \rightarrow \infty}\left\|u_{n_{k}}\right\|\right) \\
& =\lim _{k \rightarrow \infty} G\left(\left\|u_{n_{k}}\right\|\right) \\
& =\lim _{k \rightarrow \infty} F\left(u_{n_{k}}\right) \\
& =\liminf _{n \rightarrow \infty} F\left(u_{n}\right) .
\end{aligned}
$$

Hence $F$ is weakly lower semicontinuous. To show that $F$ has property $(P)$ we use the definition. Let $\left\{v_{n}\right\}$ be such a sequence, then, since $\left\|v_{n}\right\| \rightarrow \infty$, we have that

$$
\limsup _{n \rightarrow \infty} F\left(v_{n}\right)=1>F(0)
$$

i.e. (2.5) holds and hence $F$ has property $(P)$. Since $V$ is infinite dimensional, we may choose a sequence $\left\{u_{n}\right\} \subset V,\left\|u_{n}\right\|=1$ such that it contains no convergent subsequences. Let $v_{n}=n u_{n}$, then $\left\|v_{n}\right\| \rightarrow \infty$. By passing to a subsequence, we may assume that

$$
u_{n}=\frac{v_{n}}{\left\|v_{n}\right\|} \rightarrow w
$$

Also, since $F$ is bounded all the conditions of proposition 2.2 hold, yet $u_{n}=\frac{v_{n}}{\left\|v_{n}\right\|}$ does not converge to $w$.

We shall give a useful consequence of proposition 2.2 which yields property $(P)$ for functionals $F$ which have the property that the associated $\varphi$ is coercive off its zero set which is assumed a finite dimensional linear space. We prove the following.

Proposition 2.3. Suppose that $\varphi$ is nonnegative and positive homogeneous of degree $p>1$. Further assume that

$$
\operatorname{ker} \varphi=\{u: \varphi(u)=0\}
$$

is a finite dimensional subspace of $V$ such that

$$
\varphi(u+v)=\varphi(v), \forall v \in V, \forall u \in \operatorname{ker} \varphi .
$$

Furthermore assume that $V=\operatorname{ker} \varphi \oplus X$, where $X$ is a closed subspace of $V$ and $\left.\varphi\right|_{X}$ is coercive in the sense that there exists $c>0$ such that

$$
\varphi(v) \geq c\|v\|^{p}, \forall v \in X
$$

Then $F=\varphi+j$ satisfies property $(P)$ for any $j$ satisfying our stated assumptions.

Proof. We shall show that the conditions of proposition 2.2 are met. To this end let $\left\{v_{n}\right\}$ be a sequence satisfying

and

$$
\left\|v_{n}\right\| \rightarrow \infty, \quad \frac{v_{n}}{\left\|v_{n}\right\|} \rightarrow w
$$

$$
\sup F\left(v_{n}\right)<+\infty .
$$

Since $j$ is convex and lower semicontinuous, there exist constants $a, b \in \mathbb{R}$ such that

$$
j(u) \geq a\|u\|+b, \forall u \in V .
$$


Hence

Hence

$$
\begin{aligned}
0 & \geq \limsup _{n \rightarrow \infty} \frac{1}{\left\|v_{n}\right\|^{p}}\left[\varphi\left(v_{n}\right)+j\left(v_{n}\right)\right] \\
& \geq \limsup _{n \rightarrow \infty} \frac{\varphi\left(v_{n}\right)}{\left\|v_{n}\right\|^{p}}+\liminf _{n \rightarrow \infty} \frac{a\left\|v_{n}\right\|+b}{\left\|v_{n}\right\|^{p}} \\
& =\limsup _{n \rightarrow \infty} \varphi\left(\frac{v_{n}}{\left\|v_{n}\right\|}\right) \\
& =\limsup _{n \rightarrow \infty} \varphi\left(w_{n}\right) \\
& \geq \liminf _{n \rightarrow \infty} \varphi\left(w_{n}\right) \\
& \geq \varphi(w) \geq 0 .
\end{aligned}
$$

$$
w \in \operatorname{ker} \varphi \text { and } \lim _{n \rightarrow \infty} \varphi\left(w_{n}\right)=0 .
$$

It follows from (2.6) that $v_{n}=\xi_{n}+\eta_{n}, \xi_{n} \in \operatorname{ker} \varphi, \eta_{n} \in X$. Hence (2.4) and (2.5) imply

$$
\varphi\left(v_{n}\right)=\varphi\left(\eta_{n}\right) \geq c\left\|\eta_{n}\right\|^{p}
$$

and

and we conclude by $(2.9)$ that

$$
\varphi\left(w_{n}\right)=\frac{\varphi\left(v_{n}\right)}{\left\|v_{n}\right\|^{p}} \geq c\left(\frac{\left\|\eta_{n}\right\|}{\left\|v_{n}\right\|}\right)^{p}=c\left\|\frac{\eta_{n}}{\left\|v_{n}\right\|}\right\|^{p}
$$

$$
\frac{\eta_{n}}{\left\|v_{n}\right\|} \rightarrow 0
$$

Since $w_{n} \rightarrow 0$, it follows from $(2.8)$ that $\frac{\xi_{n}}{\left\|v_{n}\right\|} \rightarrow 0$. Since $\operatorname{ker} \varphi$ is finite dimensional, we conclude that $\frac{\xi_{n}}{\left\|v_{n}\right\|} \rightarrow 0$. We hence conclude that $F$ has property $(P)$.

Proposition 2.4. Let $V$ be a Hilbert space and let $K$ be a closed convex subset of $V$, $S \subset K, 0 \in K$, and assume that $A$ is nonnegative, i.e. $\langle A u, u\rangle \geq 0, u \in K$, and assume that: If $\left\{v_{n}\right\}$ is a sequence in $K$ such that

$$
\left\|v_{n}\right\| \rightarrow \infty, \quad \frac{v_{n}}{\left\|v_{n}\right\|} \rightarrow 0,
$$

and

$$
\frac{\varphi\left(v_{n}\right)}{\left\|v_{n}\right\|^{2}} \rightarrow 0
$$

then

$$
\limsup _{n \rightarrow \infty} \frac{1}{\left\|v_{n}\right\|} F\left(v_{n}\right)>0 .
$$

Then $F$ has property $(P)$ on $S$.

P r o of. Let $\left\{u_{n}\right\} \subset S$ be a sequence such that (2.4) holds, then since $\left\{u_{n}\right\} \subset K$ and (2.10) is valid we get by assumption that (2.11) is true. Since $\left\|v_{n}\right\| \rightarrow \infty$ we must have that

$$
\limsup _{n \rightarrow \infty} F\left(v_{n}\right)=\infty
$$


and therefore

$$
\limsup _{n \rightarrow \infty} F\left(v_{n}\right)>F(v),
$$

for all $v \in S$ with $j(v)<+\infty$; hence (2.5) holds.

The property described in proposition 2.4 is the property called $P$-coerciveness used in [ASV3], which in turn is an extension of some earlier properties used in [ASV1-2].

Corollary 2.5. Let $V, K$ be as above and assume again that $A$ is nonnegative and there exist $c>0, P_{0}, P_{1}: V \rightarrow \mathbb{R}_{+}$such that for all $v \in K$ satisfying

$$
\|v\|=1, \lambda v \in K, \lambda \geq 1
$$

one has

$$
\frac{1}{\lambda}\langle A(\lambda v), v\rangle+P_{0}(v)+P_{1}(v)+j^{+}(v) \geq c
$$

$\left(j^{+}(v)=\max \{j(v), 0\}\right), P_{0}(K)$ is bounded and there exists $s>0$ such that

$$
P_{0}(\lambda u) \leq \lambda^{s} P_{0}(u), \quad \forall \lambda \in[0,1], \quad \forall u \in K,
$$

further

$$
P_{1}\left(v_{n}\right) \rightarrow 0, \quad \text { whenever } v_{n} \rightarrow 0,\left\{v_{n}\right\} \subset K .
$$

Then $F$ satisfies property $(P)$.

Proof. The above result is from [ASV3], where it is shown that the above conditions imply the hypotheses of proposition 2.4 and hence property $(P)$ holds.

Corollary 2.5 contains exte nsions of the concepts compact coerciveness used in [BGT], $[\mathrm{GT}]$.

If it is the case that $V$ is a Hilbert space and the functional $F$ does not have the special structure indicated above but is only assumed to be weakly lower semicontinuous another property appears more convenient, namely we use the following definition (see [LS2]).

Definition We say that the functional $F$ has property $\left(P^{\prime}\right)$ on $S$ whenever the following hold:

If $\left\{v_{n}\right\} \subset S$ is any sequence in $S$ satisfying, as $n \rightarrow \infty$,

$$
\begin{cases}\bullet & \left\|v_{n}\right\| \rightarrow \infty \\ \bullet & w_{n}=\frac{v_{n}}{\left\|v_{n}\right\|} \rightarrow 0 \\ \bullet & \limsup _{n \rightarrow \infty} \frac{F\left(v_{n}\right)}{\left\|v_{n}\right\|^{\lambda}} \leq 0, \forall \lambda>0,\end{cases}
$$

then there exists $v_{0} \in S$ such that

$$
\limsup _{n \rightarrow \infty} F\left(v_{n}\right)>F\left(v_{0}\right)
$$

One immediately sees that if $F$ has the special structure assumed earlier and $F$ satisfies property $\left(P^{\prime}\right)$, the $F$ also satisfies property $(P)$, one only needs to observe that 
the convexity of $j$ implies that if $\left\{v_{n}\right\}$ is a sequence in $S$ such that

$$
\left\|v_{n}\right\| \rightarrow \infty
$$

then

$$
\liminf _{n \rightarrow \infty} \frac{j\left(v_{n}\right)}{\left\|v_{n}\right\|^{2}} \geq 0 .
$$

Also if $F$ is as in propositions 2.2, 2.3, 2.4 and corollary 2.5, then $F$ in fact satisfies property $\left(P^{\prime}\right)$.

3. Minimizing functionals. In this section we shall give a typical theorem for the existence of a minimum for a functional satisfying the earlier assumptions and property $(P)$. For more general results and several additional applications we refer to [LS1-2]. We shall also simplify matters by assuming that $V$ is a Hilbert space.

Associated with the functional $j$ we have the following functional $j_{\infty}: V \rightarrow \mathbb{R} \cup\{\infty\}$ defined by $j$

$$
j_{\infty}(w)=\lim _{t \rightarrow \infty} \frac{1}{t} j(t w) .
$$

This functional has the following properties as stated and proved in [ASV1].

(1) $j_{\infty}$ is convex and lower semicontinuous with $j_{\infty}(0)=0$,

(2) $j(u+v) \leq j(u)+j_{\infty}(v), \forall u, v \in H$,

(3) $j_{\infty}(\lambda w)=\lambda j_{\infty}(w) \forall \lambda \geq 0, \forall w \in V$,

(4) If $\left\|u_{n}\right\| \rightarrow \infty$ and $\frac{u_{n}}{\left\|u_{n}\right\|} \rightarrow w$, then

$$
j_{\infty}(w) \leq \liminf _{n \rightarrow \infty} \frac{j\left(u_{n}\right)}{\left\|u_{n}\right\|} .
$$

THEOREM 3.1. Let $F$ satisfy property $(P)$ on $S$ and suppose the following compatibility condition is satisfied: If $w \in V$ is such that there exists a sequence $\left\{u_{n}\right\} \subset V$ such that

$$
\begin{cases}\bullet & \left\|u_{n}\right\| \rightarrow \infty, \quad w_{n}=\frac{u_{n}}{\left\|u_{n}\right\|} \rightarrow w \\ \bullet & \limsup _{n \rightarrow \infty} \frac{1}{\left\|u_{n}\right\|} \varphi\left(u_{n}\right)+j_{\infty}(w) \leq 0 \\ \bullet \quad & \lim _{n \rightarrow \infty} \frac{\psi\left(u_{n}\right)}{\left\|u_{n}\right\|^{\lambda}}=0, \quad \forall \lambda>0,\end{cases}
$$

then we have

$$
\left\{\begin{aligned}
u-w \in S, & \forall u \in S \\
F(u-w) \leq F(u), & \forall u \in S .
\end{aligned}\right.
$$

Under the above assumptions the minimization problem (1.1) has a solution $u \in S$.

P r o of. We employ the method of elliptic regularization (see e.g. [KS]), i.e. we consider the perturbed functionals

$$
F_{\epsilon}(u)=F(u)+\epsilon\|u\|^{2}, u \in V, \epsilon>0
$$

and show that the problems

$$
F_{\epsilon}(u)=\min _{u \in S} F_{\epsilon}(v)
$$


have solutions $\left\{u_{\epsilon}\right\} \subset S$. We next show that the family $\left\{u_{\epsilon}\right\}$ is uniformly bounded, from which the result will follow using the argument presented at the beginning.

Thus consider problem (3.4). Since $j$ is lower semicontinuous and convex, there exists $\ell \in V$ and $c \in \mathbb{R}$ such that

$$
j(u) \geq\langle\ell, u\rangle+c, \quad \forall u \in V,
$$

Hence

since $0 \leq \beta<2$ we obtain immediately that

$$
\begin{aligned}
F_{\epsilon}(u) & =\epsilon\|u\|^{2}+\langle A u, u\rangle+j(u) \\
& \geq \epsilon\|u\|^{2}-c\|u\|^{\beta}-|\langle\ell, u\rangle|-|c|,
\end{aligned}
$$

$$
F_{\epsilon}(u) \rightarrow \infty \quad \text { as } \quad\|u\| \rightarrow \infty,
$$

i.e. $F_{\epsilon}$ is coercive on $V$, hence on $S$. Since $S$ is weakly closed in $V$, there exists $u_{\epsilon} \in S$ such that

$$
F_{\epsilon}\left(u_{\epsilon}\right)=\min _{v \in S} F_{\epsilon}(v) .
$$

It suffices therefore to show that $\left\{u_{\epsilon}\right\}$ is bounded. Suppose this is not the case. Then there exists a subsequence

$$
\left\{u_{n}=u_{\epsilon_{n}}\right\} \subset\left\{u_{\epsilon}\right\}, \quad \epsilon_{n} \downarrow 0,
$$

such that

$$
\left\|u_{n}\right\| \rightarrow \infty, \quad n \rightarrow \infty
$$

It follows from (3.5) that for all $v \in S$

$$
F\left(u_{n}\right)+\epsilon_{n}\left\|u_{n}\right\|^{2} \leq F(v)+\epsilon_{n}\|v\|^{2} .
$$

Hence

$$
\begin{aligned}
\limsup _{n \rightarrow \infty} \frac{1}{\left\|u_{n}\right\|} F\left(u_{n}\right) & \leq \limsup _{n \rightarrow \infty} \frac{1}{\left\|u_{n}\right\|}\left(F\left(u_{n}\right)+\epsilon_{n}\left\|u_{n}\right\|^{2}\right) \\
& \leq \limsup _{n \rightarrow \infty} \frac{1}{\left\|u_{n}\right\|}\left(F(v)+\epsilon_{n}\|v\|^{2}\right) \\
& =0 .
\end{aligned}
$$

Passing to a subsequence, if necessary, we may assume that

$$
w_{n}=\frac{u_{n}}{\left\|u_{n}\right\|} \rightarrow w .
$$

Using the properties of the functional $j_{\infty}$ (see above) we have

hence

$$
j_{\infty}(w) \leq \liminf _{n \rightarrow \infty} \frac{j\left(u_{n}\right)}{\left\|u_{n}\right\|},
$$

$$
\begin{aligned}
& \limsup _{n \rightarrow \infty} \frac{1}{\left\|u_{n}\right\|} \varphi\left(u_{n}\right)+j_{\infty}(w) \leq \\
& \limsup _{n \rightarrow \infty} \frac{1}{\left\|u_{n}\right\|} \varphi\left(u_{n}\right)+\liminf _{n \rightarrow \infty} \frac{j\left(u_{n}\right)}{\left\|u_{n}\right\|} \leq \\
& \limsup _{n \rightarrow \infty} \frac{1}{\left\|u_{n}\right\|} F\left(u_{n}\right) \leq 0 .
\end{aligned}
$$


Further, since $\left\{u_{n}\right\} \subset S, \psi\left(u_{n}\right)=\gamma$, therefore we must have

$$
\limsup _{n \rightarrow \infty} \frac{\psi\left(u_{n}\right)}{\left\|u_{n}\right\|^{\lambda}}=0, \quad \lambda>0 .
$$

Thus (3.1) holds and consequently, by hypothesis, (3.2) must hold. In particular,

$$
u_{n}-w \in S, \quad F\left(u_{n}-w\right) \leq F\left(u_{n}\right), \quad \forall n .
$$

Letting $v=u_{n}-w$ in (3.6), we get

$$
\begin{aligned}
F\left(u_{n}\right)+\epsilon_{n}\left\|u_{n}\right\|^{2} & \leq F\left(u_{n}-w\right)+\epsilon_{n}\left\|u_{n}-w\right\|^{2} \\
& \leq F\left(u_{n}\right)+\epsilon_{n}\left\|u_{n}-w\right\|^{2},
\end{aligned}
$$

thus

$$
\left\|u_{n}\right\|^{2} \leq\left\|u_{n}-w\right\|^{2}, \quad \forall n
$$

or

$$
\left\|u_{n}\right\|^{2} \leq\left\|u_{n}\right\|^{2}-2\left\langle u_{n}, w\right\rangle+\|w\|^{2}
$$

and hence

$$
\left\langle u_{n}, w\right\rangle \leq \frac{1}{2}\|w\|^{2}
$$

Therefore

$$
\left\langle w_{n}, w\right\rangle=\frac{1}{\left\|u_{n}\right\|}\left\langle u_{n}, w\right\rangle \leq \frac{\|w\|^{2}}{2\left\|u_{n}\right\|},
$$

and since $w_{n} \rightarrow w$ we conclude $w=0$, i.e.

$$
w_{n} \rightarrow 0 \text {. }
$$

Using (3.6) again and dividing the inequality by $\left\|u_{n}\right\|^{2}$, we obtain

i.e.

$$
\limsup _{n \rightarrow \infty} \frac{1}{\left\|u_{n}\right\|^{2}} F\left(u_{n}\right) \leq 0
$$

$$
\limsup _{n \rightarrow \infty}\left\{\frac{1}{\left\|u_{n}\right\|^{2}} \varphi\left(u_{n}\right)+\frac{1}{\left\|u_{n}\right\|^{2}} j\left(u_{n}\right)\right\} \leq 0 .
$$

Since $j$ is convex and $j(0)=0$, we have

$$
\begin{aligned}
\liminf _{n \rightarrow \infty} \frac{j\left(u_{n}\right)}{\left\|u_{n}\right\|^{2}} & \geq \liminf _{n \rightarrow \infty} j\left(\frac{u_{n}}{\left\|u_{n}\right\|^{2}}\right) \\
& \geq j\left(\lim _{n \rightarrow \infty} \frac{u_{n}}{\left\|u_{n}\right\|^{2}}\right)=0 .
\end{aligned}
$$

Hence (3.7) implies

$$
\limsup _{n \rightarrow \infty} \frac{1}{\left\|u_{n}\right\|^{2}} \varphi\left(u_{n}\right) \leq 0 .
$$

We thus have all three conditions in the definition of property $(P)$ holding, consequently there must exist $u_{0} \in S$ such that

$$
\limsup _{n \rightarrow \infty} F\left(u_{n}\right)>F\left(u_{0}\right) .
$$

But (3.6) implies

$$
F\left(u_{n}\right) \leq F_{\epsilon_{n}}\left(u_{n}\right) \leq F_{\epsilon_{n}}\left(u_{0}\right)=F\left(u_{0}\right)+\epsilon_{n}\left\|u_{0}\right\|^{2},
$$

and we obtain a contradiction to (3.8). 
4. Critical point theorems. We now show how theorem 3.1 together with Liusternik's theorem (Lagrange multipliers, cf. [K]) may be used to obtain results about critical points of certain functionals. Much of the discussion in the first part follows [LS1].

We assume that $V$ is a Hilbert space and

$$
a: V \times V \rightarrow \mathbb{R}
$$

is a continuous bilinear form which is positive, i.e.

$$
a(u, u) \geq 0, \quad \forall u \in V,
$$

then

$$
\varphi: V \rightarrow \mathbb{R}
$$

given by

$$
\varphi(u)=a(u, u)
$$

is a nonnegative weakly lower semicontinuous functional on $V$. Let us assume that

$$
\operatorname{dim} W=\operatorname{dim} \operatorname{ker} a=\operatorname{dim}\{u: a(u, u)=0\}<+\infty,
$$

and there exists $c>0$ such that

$$
\varphi(u) \geq c\|u\|^{2}, \quad \forall u \in W^{\perp},
$$

i.e. $\varphi$ is coercive on $W^{\perp}$. Let

$$
\psi: V \rightarrow \mathbb{R}
$$

be a weakly continuous functional homogeneous of degree $\alpha>1, \alpha \neq 2$, i.e.

$$
\psi(\lambda u)=\lambda^{\alpha} \psi(u), \quad \forall \lambda \geq 0, u \in H
$$

and let $S$ be given by

$$
S=\{u \in H: \psi(u)=\gamma\},
$$

which we assume to be nonempty.

We have the following consequence of theorem 3.1

Corollary 4.1. Let $\varphi$ and $\psi$ satisfy the above conditions and assume

$$
\psi(u) \neq 0, \quad \forall u \in(W=\operatorname{ker} a) \backslash\{0\} .
$$

Then there exists $u \in S$ such that

$$
\varphi(u)=\min _{v \in S} \varphi(v)
$$

Pro of. We check the conditions of theorem 3.1. To this end we let

$$
\langle A u, v\rangle=a(u, v)
$$

and $j \equiv 0$. That $F=\varphi$ satisfies property $(P)$ follows from proposition 2.3 .

We next check the compatibility condition of theorem 3.1. To this end, let $\left\{u_{n}\right\},\left\{w_{n}\right\}$, and $w$ satisfy (3.1). We shall show that $w=0$ and hence (3.2) will obviously hold. We have (since $\left.j_{\infty} \equiv 0\right)$

$$
\limsup _{n \rightarrow \infty} \frac{1}{\left\|u_{n}\right\|} a\left(u_{n}, u_{n}\right) \leq 0
$$


(see $(3.1))$, hence

$$
\begin{aligned}
\limsup _{n \rightarrow \infty} a\left(w_{n}, w_{n}\right) & =\limsup _{n \rightarrow \infty} \frac{1}{\left\|u_{n}\right\|^{2}} a\left(u_{n}, u_{n}\right) \\
& \leq 0 .
\end{aligned}
$$

Since $\varphi$ is weakly lower semicontinuous

$$
\varphi(w) \leq \liminf _{n \rightarrow \infty} \varphi\left(w_{n}\right) \leq 0
$$

and therefore

$$
\varphi(w)=a(w, w)=0,
$$

i.e. $w \in W=\operatorname{ker} a$. Now using (3.1) for $\psi$ and the weak continuity of $\psi$, we get

$$
\begin{aligned}
0=\lim _{n \rightarrow \infty} \frac{1}{\left\|u_{n}\right\|^{\alpha}} \psi\left(u_{n}\right) & =\lim _{n \rightarrow \infty} \psi\left(\frac{u_{n}}{\left\|u_{n}\right\|}\right) \\
& =\lim _{n \rightarrow \infty} \psi\left(w_{n}\right)=\psi(w),
\end{aligned}
$$

which by hypothesis implies that $w=0$.

Corollary 4.2. Assume the conditions of corollary 4.1 and that $\psi$ also satisfies:

$$
\left\{\begin{array}{l}
\exists u \in V \quad \text { such that } \quad \psi(u)<0 \\
\psi(u)>0, \quad \forall u \in W \backslash\{0\} .
\end{array}\right.
$$

Then the functional $f$ defined by

$$
f(u)=\varphi(u)+\psi(u)
$$

has a nontrivial critical point.

Proof. If $u \in V$ is such that $\psi(u)=M<0$, then $\psi\left(|M|^{-1 / \alpha} u\right)=-1$. Thus

$$
S=\{u: \psi(u)=-1\} \neq \emptyset .
$$

Since $\psi$ is homogeneous, we have that for $u \in S$

$$
\left\langle\psi^{\prime}(u), u\right\rangle=\alpha \psi(u)=-\alpha \neq 0,
$$

hence $\psi^{\prime}(u) \neq 0, u \in S$. Let $u$ be the minimizer of corollary 4.1, then we obtain from Liusternik's theorem (see $[\mathrm{K}]$ ) the existence of a Lagrange multiplier $\mu \in \mathbb{R}$ such that

$$
\varphi^{\prime}(u)+\mu \psi^{\prime}(u)=0
$$

Hence

$$
\begin{aligned}
0=\left\langle\varphi^{\prime}(u), u\right\rangle+\mu\left\langle\psi^{\prime}(u), u\right\rangle & =2 \varphi(u)+\mu \alpha \psi(u) \\
& =2 \varphi(u)-\mu \alpha .
\end{aligned}
$$

Since $\varphi(u)>0$ for $u \in S$, we get $\alpha>0$. Rescaling, i.e. putting $v=\mu^{\frac{1}{\alpha-2}} u$, we see that $v$ is a critical point of $f$.

Rem ark. Corollary 4.2 is a theorem from [BTW].

The following corollary contains corollary 4.1 and corollary 4.2 as special cases. 
Corollary 4.3. Let $\varphi$ and $\psi$ be as above (i.e. satisfy the condition (4.1) - (4.6)) and assume that

$$
\psi(v-u)=\psi(v), \quad \forall v \in V, \quad \forall u \in W \cap \operatorname{ker} \psi,
$$

where $\operatorname{ker} \psi=\{u: \psi(u)=0\}$, then the problem (4.8) has a solution.

Proof. We have shown above already that property $(P)$ holds. We hence must verify that (3.1) and (3.2) are true. Thus let $w,\left\{w_{n}\right\}$, and $\left\{u_{n}\right\}$ be as in these conditions. We know that $\varphi(w)=\psi(w)=0$, (see corollary 4.1), i.e. $w \in \operatorname{ker} \varphi \cap \operatorname{ker} \psi$. By hypothesis we have (4.12) holding, i.e. $\psi(v-w)=\psi(v), \forall v \in V$. Hence $v-w \in S$, whenever $v \in S$. Moreover, since $w \in \operatorname{ker} \varphi=W$, we have

$$
a(w, v)+a(v, w)=\left\langle\varphi^{\prime}(w), v\right\rangle=0, \forall v \in W
$$

hence

$$
\begin{aligned}
\varphi(v-w) & =\varphi(v)+\varphi(w)-[a(v, w)+a(w, v)] \\
& =\varphi(v)
\end{aligned}
$$

proving (3.2) and hence the result follows.

COROLlary 4.4. Suppose again that $\psi \in C^{1}$ and that $\varphi$ and $\psi$ satisfy the conditions at the beginning of this section and that

(i) $\psi(u)<0$, for some $u \in V$

(ii) $\psi(u) \geq 0, \quad \forall u \in W$

$$
\text { and }
$$

(iii) if $u \in W$ is such that $\psi(u)=0$, then $\psi(v-u)=\psi(v), \forall v \in V$.

Then the functional $f$ given by (4.10) has a critical point.

Proof. It follows from (i) that

$$
S=\{u \in V: \psi(u)=-1\} \neq \emptyset .
$$

Since $\left\langle\psi^{\prime}(u), u\right\rangle=-\alpha$, we have $\psi^{\prime}(u) \neq 0, \forall u \in S$, and we again may apply Liusternik's theorem $([\mathrm{K}])$ together with corollary 4.3 to obtain

$$
\varphi^{\prime}(u)+\mu \psi^{\prime}(u)=0
$$

for some $\mu \in \mathbb{R}$.

As in corollary 4.2 we have

$$
2 \varphi(u)-\mu \alpha=0
$$

and since $\psi \geq 0$ on $\operatorname{ker} \varphi$ we have

$$
S \cap \operatorname{ker} \varphi=\emptyset .
$$

This implies that $\varphi>0$ on $S$, hence $\varphi(u)>0$ and $\mu>0$. Again the rescaling

$$
v=\lambda^{-1} u=\mu^{\frac{1}{\alpha-2}} u
$$

yields the desired conclusion.

Now we apply theorem 3.1 for minimization problems on closed convex sets to derive existence results for constrained critical points, which are solutions of variational 
inequalities. We assume that $S$ is a closed, convex set in $V$, and $\varphi$ is

$$
F=\varphi+j+I_{S} \text {. }
$$

We consider the minimization problem:

$$
u \in V: F(u)=\min _{v \in V} F(v),
$$

which is equivalent to the following problem:

$$
u \in S:(\varphi+j)(u)=\min _{v \in S}(\varphi+j)(v) .
$$

We need the following simple lemma (cf. Sect. 3.5.4, [DL]).

LEMMA 4.5. If $u$ is a solution of (4.14) then $u$ satisfies the variational inequality:

$$
\left\{\begin{array}{l}
\left\langle\varphi^{\prime}(u), v-u\right\rangle+j(v)-j(u) \geq 0, \forall v \in S \\
u \in S .
\end{array}\right.
$$

Proof. Let $u \in V$ satisfy (4.14). Since $j(w)<\infty$ for some $w \in S$, we have $F(u)=$ $\min _{v \in V} F(v)<\infty$. Hence $u \in S$ and $j(u)<\infty$.

For $v \in S$, we have $w=u+t(v-u) \in S, \forall t \in[0,1]$, and

$$
\begin{aligned}
\varphi(u)+j(u)=F(u) & \leq F(w)=\varphi(u+t(v-u))+j(u+t(v-u)) \\
& \leq \varphi(u+t(v-u))+[(1-t) j(u)+t j(v)] .
\end{aligned}
$$

Therefore

$$
\varphi(u+t(v-u))-\varphi(u)+t[j(v)-j(u)] \geq 0, \forall t \in[0,1] .
$$

Dividing this inequality by $t \in[0,1]$ and letting $t \rightarrow 0^{+}$, we obtain (4.15).

As in [ASV1], we denote by $r c S$ the recession cone of $S$ :

$$
r c S=\{w \in V: u+t w \in S, \forall u \in S, \forall t \geq 0\} .
$$

It is proved (see e. g. $[\mathrm{R}])$ that

$$
r c S=\bigcap_{t>0} t\left(S-u_{0}\right), u_{0} \in S
$$

is a closed, convex cone in $V$, and moreover

$$
\begin{aligned}
w \in r c S & \Longleftrightarrow w+u \in S, \forall u \in S \\
& \Longleftrightarrow \exists u \in S: u+t w \in S, \forall t \geq 0 .
\end{aligned}
$$

From theorem 3.1 and lemma 4.5, we have the following existence result for (4.15):

Corollary 4.6. Let $F$ have property $(P)$ on $S$ and suppose the following compatibility condition is satisfied: If $w \in \operatorname{rcS}$ is such that there exists a sequence $\left\{u_{n}\right\} \subset S$ such that

$$
\left\{\begin{array}{l}
\bullet \quad\left\|u_{n}\right\| \rightarrow \infty, \quad \frac{u_{n}}{\left\|u_{n}\right\|} \rightarrow w \\
\bullet \quad \limsup _{n \rightarrow \infty} \frac{1}{\left\|u_{n}\right\|} \varphi\left(u_{n}\right)+j_{\infty}(w) \leq 0,
\end{array}\right.
$$

then we have $-w \in \operatorname{rcS}$, and

$$
\varphi(u-w)+j(u-w) \leq \varphi(u)+j(u), \forall u \in S .
$$

Under these assumptions, the variational inequality (4.15) has a solution. 
Proof. We check the conditions of theorem 3.1 with $F$ given by $(4.13)$ and $\psi \equiv 0$. Let $w,\left\{u_{n}\right\}$ satisfy (3.1); then (4.16) immediately holds. We prove $w \in r c S$. As in the proof of theorem 3.1, we have $u_{n} \in S, \forall n$. Let $u \in S$. Since $\left\|u_{n}\right\|>1$ for all $n$ large,one has

$$
\left(1-\frac{1}{\left\|u_{n}\right\|}\right) u+\frac{1}{\left\|u_{n}\right\|} u_{n} \in S .
$$

Letting $n \rightarrow \infty$, we obtain from (3.1) and the fact that $S$ is weakly closed that $u+w \in S$. Since this holds for all $u \in S$, we have $w \in r c S$ by (4.16). By hypothesis, we get $-w \in r c S$, and (4.18). We prove $F(u-w) \leq F(u)$ for all $u \in V$. If $u \notin S$ then $F(u)=I_{S}(u)=\infty$ and this is clearly true. If $u \in S$ then $u-w \in S$ by (4.16) and

$$
\begin{aligned}
F(u-w) & =\varphi(u-w)+j(u-w)+I_{S}(u-w)=\varphi(u-w)+j(u-w) \\
& \leq \varphi(u)+j(u)=F(u) .
\end{aligned}
$$

We have (3.2); and all conditions of theorem 3.1 are satisfied. By this theorem, (4.14) is solvable. Applying lemma 4.5, we obtain the existence of a solution of (4.15).

Re m ark. Corollary 4.6 is, in some sense, similar to Theorem 3 of [ASV3].

Existence results for noncoercive variational inequalities containing non convex functionals $j$ are considered in [LS2].

An immediate consequence of corollary 4.6 is the following:

Corollary 4.7. Assume $F$ has property $(P)$ on $S$, and that if $w \in \operatorname{rcS}$ satisfies (4.17) then $w=0$. Then (4.15) has a solution.

5. Some applications. In this section we shall consider a boundary value problem for a semilinear elliptic equation, and a quasilinear variational inequality as applications of the results derived above.

Let $\Omega$ be a bounded domain in $\mathbb{R}^{N}$ with smooth boundary. Consider the boundary value problem

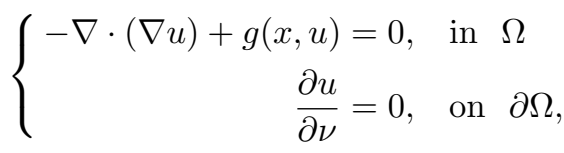

where $g: \Omega \times \mathbb{R} \rightarrow \mathbb{R}$ is a given function satisfying Carathéodory type conditions and the growth conditions given below.

This problem has the following weak formulation:

$$
\left\{\begin{array}{l}
\int_{\Omega} \nabla u \cdot \nabla v+\int_{\Omega} g(x, u) v=0, \forall v \in H^{1}(\Omega) \\
u \in H^{1}(\Omega),
\end{array}\right.
$$

where $V=H^{1}(\Omega)$ is the Sobolev space of $L^{2}$ functions having $L^{2}$ first (weak) derivatives, endowed with the usual norm $\|u\|=\|u\|_{H^{1}(\Omega)}$. Concerning $g$ we assume that it is the growth condition

$$
|g(x, u)| \leq a+b|u|^{q-1}
$$


where $a, b$ are constants and

$$
\left\{\begin{array}{r}
1<q<2^{*}=2 N(N-2)^{-1}, \text { if } 2<N \\
1<q<2^{*}=\infty, \text { if } N=2 .
\end{array}\right.
$$

Let

$$
G(x, u)=\int_{0}^{u} g(x, s) d s,
$$

then standard arguments give that solutions of (5.2) are given by the critical points of the functional

$$
f(u)=\varphi(u)+\psi(u)
$$

where

$$
\varphi(u)=\frac{1}{2} \int_{\Omega}|\nabla u|^{2}
$$

and

$$
\psi(u)=\int_{\Omega} G(x, u(x)), u \in V .
$$

It follows that $\varphi$ and $\psi$ are of class $C^{1}$ and

$$
\left\{\begin{array}{l}
\left\langle\varphi^{\prime}(u), v\right\rangle=\int_{\Omega} \nabla u \cdot \nabla v \\
\left\langle\psi^{\prime}(u), v\right\rangle=\int_{\Omega} g(x, u(x)) v, \forall u, v \in V,
\end{array}\right.
$$

where $\langle\cdot, \cdot\rangle$ denotes the pairing between $V$ and $V^{*}$, the inner product of $H^{1}$.

With this setup we have the following result.

Corollary 5.1. Assume that

$$
G(x, t u)=t^{\alpha} G(x, u), \forall t \geq 0, \text { a.e. } x \in \Omega, \forall u \in \mathbb{R},
$$

and

$$
\int_{\Omega} G(x, \pm 1)>0
$$

and

$$
\int_{\Omega} G(x, u(x))<0, \text { for some } u \in V \text {. }
$$

Then (5.2) has a nontrivial solution.

Proof. We first check that $\varphi$ has property $(P)$. It is clear that $\varphi$ is nonnegative and continuous on $V$ and $\varphi$ is convex and hence weakly lower semicontinuous. We shall employ proposition 2.2 with $F=\varphi$. Thus let $\left\{v_{n}\right\},\left\{w_{n}\right\}, w$ be as in the proposition. It 
follows that $\lim _{n \rightarrow \infty} \frac{\varphi\left(v_{n}\right)}{\left\|v_{n}\right\|^{2}}=0$, and hence

$$
\begin{aligned}
0 & \leq \varphi(w) \leq \liminf _{n \rightarrow \infty} \varphi\left(w_{n}\right) \\
& \leq \limsup _{n \rightarrow \infty} \varphi\left(w_{n}\right) \\
& =\limsup _{n \rightarrow \infty} \varphi\left(\frac{v_{n}}{\left\|v_{n}\right\|}\right) \\
& =\limsup _{n \rightarrow \infty} \frac{\varphi\left(v_{n}\right)}{\left\|v_{n}\right\|^{2}}=0 .
\end{aligned}
$$

Hence $\varphi(w)=0$, i.e. $w=$ constant. Thus also

$$
\int_{\Omega}\left|\nabla\left(w_{n}-w\right)\right|^{2} \rightarrow 0
$$

which together with the compactness of the embedding $V \hookrightarrow L^{2}(\Omega)$ implies that $w_{n} \rightarrow w$. Thus $\varphi$ has property $(P)$.

The above also shows that

$$
\operatorname{ker} \varphi=\mathbb{R}, \varphi(v-u)=\varphi(v), \forall v \in V, \forall u \in \operatorname{ker} \varphi .
$$

The growth conditions on $g$ and (5.3) imply that the embedding $V \hookrightarrow L^{q}(\Omega)$ is compact and thus if $u_{n} \rightarrow u$, then $u_{n} \rightarrow u$ in $L^{q}(\Omega)$ and hence $G\left(\cdot, u_{n}\right) \rightarrow G(\cdot, u)$ in $L^{1}(\Omega)$, which implies the complete continuity of $\psi$. Using the other hypotheses on $g$ we may now employ part $(b)$ of corollary 4.1 to complete the proof.

A particular choice of $g$ is

$$
g(x, u)=k(x)|u|^{\alpha-2} u
$$

where $k \in C(\bar{\Omega}), 1<2^{*} \alpha, \alpha \neq 2$. In this case the Carathéodory conditions are easily verified. The other conditions of the corollary will hold whenever $k$ changes sign on $\Omega$ and $\int_{\Omega} k>0$.

For examples of the type just discussed for more general quasilinear problems, like the $p$-Laplacian, we refer to [LS1-2].

The next example is for the existence of solutions of the following quasilinear variational inequality:

$$
\left\{\begin{array}{l}
\int_{\Omega} a(u) \nabla u \nabla(v-u)+J(v)-J(u) \geq \int_{\Omega} f(v-u), \forall v \in S \\
u \in S .
\end{array}\right.
$$

Here $\Omega, V$ are as in the above example, $f \in L^{2}(\Omega)$, and

$$
a(u)=\frac{2|\nabla u|^{p}}{1+|\nabla u|^{p}}+\frac{p|\nabla u|^{p}}{\left(1+|\nabla u|^{p}\right)^{2}}, u \in H^{1}(\Omega),
$$

where $p$ is a given number, $0 \leq p \leq 7$. We assume

$$
S=\{u \in V: u \geq \zeta \text { a. e. on } \Omega\}
$$

and

$$
J(u)=\int_{\Omega} \psi(u(x)) d x, u \in H^{1}(\Omega),
$$


where $\zeta: \Omega \rightarrow \mathbb{R}, \psi: \mathbb{R} \rightarrow \mathbb{R}_{+}$are given functions, $\zeta \in L^{\infty}(\Omega)$ and $\psi$ is convex, continuous such that $\psi(0)=0$. It can be checked that $S$ is a closed, convex set in $V=H^{1}(\Omega)$, and $J$ is a convex, lower continuous functional from $V$ to $[0, \infty]$. Since

$$
u \mapsto \int_{\Omega} f u, u \in V
$$

is a linear bounded functional, $j$ given by

$$
j(u)=J(u)-\int_{\Omega} f u, u \in V
$$

is convex and lower semicontinuous from $V$ to $\mathbb{R} \cup\{\infty\}$. One can show from the convexity of $\psi$ that the limit

$$
\psi^{+}=\lim _{t \rightarrow \infty} \frac{\psi(t)}{t}
$$

exists in $\mathbb{R} \cup\{\infty\}$. As usual, $|\Omega|$ denotes the Lebesgue measure of $\Omega$. We have the following result:

Corollary 5.2. If

$$
\frac{1}{|\Omega|} \int_{\Omega} f(x) d x<\psi^{+}
$$

then (5.8) has a solution in $H^{1}(\Omega)$.

Proof. Let $A: V \rightarrow V^{*}, \varphi: V \rightarrow \mathbb{R}$ be given by

$$
\begin{gathered}
\langle A u, v\rangle=\int_{\Omega} \frac{|\nabla u|^{p}}{1+|\nabla u|^{p}} \nabla u \nabla v, \\
\varphi(u)=\langle A u, u\rangle=\int_{\Omega} \frac{|\nabla u|^{p+2}}{1+|\nabla u|^{p}}, \forall u, v \in V .
\end{gathered}
$$

Since

$$
\int_{\Omega}\left|\frac{|\nabla u|^{p}}{1+|\nabla u|^{p}} \nabla u \nabla v\right| \leq \int_{\Omega}|\nabla u||\nabla v| \leq\|u\|\|v\|, \forall u, v \in V,
$$

$A$ and $\varphi$ are well defined. Moreover, it is directly verified that the function $\xi \mapsto \frac{|\xi|^{p+2}}{1+|\xi|^{p}}, \xi \in$ $\mathbb{R}^{N}$ is of class $C^{1}$ and convex on $\mathbb{R}^{N}$ (if and only if $p \leq 7$ (as may easily be checked using Maple)) with

$$
\nabla\left(\frac{|\xi|^{p+2}}{1+|\xi|^{p}}\right)=\frac{|\xi|^{p}\left[p+2\left(1+|\xi|^{p}\right)\right] \xi}{\left(1+|\xi|^{p}\right)^{2}}, \xi \in \mathbb{R}^{N} .
$$

We have $\varphi \in C^{1}(V, \mathbb{R}), \varphi$ is convex on $V$, and

$$
\left\langle\varphi^{\prime}(u), v\right\rangle=\int_{\Omega} a(u) \nabla u \nabla v, \forall u, v \in V .
$$

Therefore (5.8) is of the form (4.15). Let $F$ be given by (4.13). Since $\varphi$ is convex and continuous, it is weakly lower semicontinuous It follows that $F$ also has this property. We now check that $F$ has property $(P)$ on $V$ by using proposition 2.4. Let $\left\{v_{n}\right\} \subset V$ satisfy the conditions of this proposition. Suppose that (2.11) does not hold, i.e.,

$$
\limsup _{n \rightarrow \infty} \frac{F\left(v_{n}\right)}{\left\|v_{n}\right\|} \leq 0 \text {. }
$$


Since $w_{n}=v_{n} /\left\|v_{n}\right\| \rightarrow 0$ in $L^{2}(\Omega)$, we have

$$
\lim _{n \rightarrow \infty} \frac{1}{\left\|v_{n}\right\|} \int_{\Omega} f v_{n}=\lim _{n \rightarrow \infty} \int_{\Omega} f w_{n}=0 .
$$

Moreover, since $\varphi, J$, and $I_{S}$ are non negative on $V$, it follows from (5.12) that

$$
\lim _{n \rightarrow \infty} \frac{\varphi\left(v_{n}\right)}{\left\|v_{n}\right\|}=0
$$

We have on the other hand

$$
\begin{aligned}
\frac{\varphi\left(v_{n}\right)}{\left\|v_{n}\right\|} & =\int_{\Omega} \frac{\left|\nabla\left(v_{n} /\left\|v_{n}\right\|\right)\right|^{p+2}\left\|v_{n}\right\|^{p+1}}{1+\left|\nabla v_{n}\right|^{p}} \\
& \geq\left\|v_{n}\right\| \int_{\Omega} \frac{\left|\nabla w_{n}\right|^{p+2}}{\left\|v_{n}\right\|^{-p}+\left|\nabla w_{n}\right|^{p}} \\
& \geq \int_{\Omega} \frac{\left|\nabla w_{n}\right|^{p+2}}{1+\left|\nabla w_{n}\right|^{p}}
\end{aligned}
$$

for all $n$ sufficiently large (such that $\left\|v_{n}\right\| \geq 1$ ). Hence

$$
\lim _{n \rightarrow \infty} \int_{\Omega} \frac{\left|\nabla w_{n}\right|^{p+2}}{1+\left|\nabla w_{n}\right|^{p}}=0 .
$$

Putting $\Omega_{n}=\left\{x \in \Omega:\left|\nabla w_{n}(x)\right| \geq 1\right\}, n=1,2, \ldots$, one has

$$
\frac{\left|\nabla w_{n}\right|^{p+2}}{1+\left|\nabla w_{n}\right|^{p}} \geq\left\{\begin{array}{l}
\frac{1}{2}\left|\nabla w_{n}(x)\right|^{2} \quad \text { if } \quad x \in \Omega_{n}, \\
\frac{1}{2}\left|\nabla w_{n}(x)\right|^{p+2} \quad \text { if } \quad x \in \Omega \backslash \Omega_{n} .
\end{array}\right.
$$

Therefore

$$
\lim _{n \rightarrow \infty} \int_{\Omega_{n}}\left|\nabla w_{n}(x)\right|^{2}=\lim _{n \rightarrow \infty} \int_{\Omega \backslash \Omega_{n}}\left|\nabla w_{n}(x)\right|^{p+2}=0 .
$$

Since $p \geq 0$, by Hölder's inequality,

$$
\int_{\Omega \backslash \Omega_{n}}\left|\nabla w_{n}(x)\right|^{2} \leq|\Omega|^{\frac{p}{p+2}}\left(\int_{\Omega \backslash \Omega_{n}}\left|\nabla w_{n}(x)\right|^{p+2}\right)^{\frac{2}{p+2}} .
$$

Hence $\lim _{n \rightarrow \infty} \int_{\Omega}\left|\nabla w_{n}(x)\right|^{2}=0$. As $w_{n} \rightarrow 0$ in $L^{2}(\Omega)$, we have from this limit that $w_{n} \rightarrow 0$ in $H^{1}(\Omega)$, contradicting the fact that $\left\|w_{n}\right\|=1, \forall n$. This proves (2.11) and therefore $F$ has property $(P)$.

Now, let $w,\left\{u_{n}\right\}$ satisfy (4.17). Since $f$ is linear, we have $f_{\infty}=f$. Hence $j_{\infty}=J_{\infty}-f$. From (4.17), we get

$$
\limsup _{n \rightarrow \infty} \frac{\varphi\left(u_{n}\right)}{\left\|u_{n}\right\|}+J_{\infty}(w) \leq \int_{\Omega} f w .
$$

Since $J_{\infty} \geq 0$, this inequality implies that

Hence

$$
\limsup _{n \rightarrow \infty} \frac{\varphi\left(u_{n}\right)}{\left\|u_{n}\right\|^{2}} \leq 0
$$

$$
\lim _{n \rightarrow \infty} \frac{\varphi\left(u_{n}\right)}{\left\|u_{n}\right\|^{2}}=0
$$


Using arguments as in the proof of the property $(P)$ of $F$, one can conclude from (5.14) that

$$
\lim _{n \rightarrow \infty} \int_{\Omega}\left|\nabla w_{n}(x)\right|^{2}=0, \quad\left(w_{n}=u_{n} /\left\|u_{n}\right\|\right) .
$$

Since $u \mapsto \int_{\Omega}|\nabla u|^{2}$ is weakly lower semicontinuous on $H^{1}(\Omega)$, we have

$$
0 \leq \int_{\Omega}|\nabla w|^{2} \leq \liminf _{n \rightarrow \infty} \int_{\Omega}\left|\nabla w_{n}(x)\right|^{2}=0 .
$$

Hence $\nabla w=0$ a. e. in $\Omega$, i. e., $w=$ constant.

On the other hand, it follows from (5.9) that

$$
r c S=\{u \in V: u \geq 0 \text { a. e. on } \Omega\} .
$$

Thus $w \geq 0$ a. e. on $\Omega$, that is $w \in \mathbb{R}_{+}$. Now,

$$
\begin{aligned}
J_{\infty}(w) & =\lim _{t \rightarrow \infty} \int_{\Omega} \frac{\psi(t w)}{t} \\
& =\lim _{t \rightarrow \infty}|\Omega| \frac{\psi(t w)}{t w} w \\
& = \begin{cases}|\Omega| \psi^{+} w & \text { if } w>0 \\
0 & \text { if } w=0 .\end{cases}
\end{aligned}
$$

From (5.13), we have that

$$
J_{\infty}(w) \leq \int_{\Omega} f w=w \int_{\Omega} f .
$$

From (5.11) and (5.15), we see that this inequality happens only if $w=0$. Our conclusion now follows from corollary 4.7 .

\section{References}

[ASV1] D. D. Ang, K. Schmitt and L. K. Vy, Noncoercive variational inequalities: some applications, Nonlinear Analysis, TMA 15(1990), 497-512.

[ASV2] D. D. Ang, K. Schmitt and L. K. Vy, Variational inequalities and the contact of elastic plates, in: Differential Equations and Applications in Biology, Physics, and Engineering, Goldstein, Kappel, Schappacher (eds.), M. Dekker, N.Y., 1991,1-19.

[ASV3] D.D. Ang, K. Schmitt and L. K. Vy, P-coercive variational inequalities and unilateral problems for von Karman's equations, in: World Scientific Series in Applied Math., vol. 1, R. Agarwal (ed.), World Scientific, Singapore, 1992,15-29.

[AV] D. D. Ang and L. K. Vy, Some applications and extensions of P-coercive variational inequalities, preprint(1993).

[BGT] G. Baiocchi, F. Gastaldi and F. Tomarelli, Inéquations variationnelles non coercives, C. R. Acad. Sc. Paris 299 (1984), 647-650.

[BTW] A.BenNaoum, C. Troestler and M. Willem, Existence and multiplicity results for homogeneous second order differential equations, J. Diff. Equations 112 (1994), 239-249.

[B] V. Brézis, Analyse Fonctionnelle, Masson, Paris 1983. 
[DL] G. Duvaut and J. L. Lions, Les Inéquations en Mécanique et en Physique, Dunod, Paris, 1972.

[F] G. Fichera, Problemi elastostatici con vincoli unilaterali: il problema di Signorini con ambigue condizioni al contorno, Mem. Acc. Naz. Lincei, Ser. 8 vol 7 (1964), 91-140.

[GT] F. Gastaldi and F. Tomarelli, Some remarks on nonlinear and noncoercive variational inequalities, Boll. U. M. I. (7) 1-B (1987), 143-165.

[H] P. Hess, On nonlinear mappings of monotone type with respect to Banach spaces, Ind. Univ. Math. J. 23 (1974), 645-654.

[J] R. C. James, Orthogonality and linear functionals in normed spaces, Trans. Amer. Math. Soc. 61 (1947), 265-292.

[KS] D. Kinderlehrer and G. Stampacchia, Introduction to Variational Inequalities, Academic Press, New York, 1980.

[K] M. A. Krasnosel'skii, Topological Methods in the Theory of Nonlinear Integral Equations, Pergamon, New York, 1964.

[LS] J. L. Lions and G. Stampacchia, Variational inequalities, Comm. Pure Appl. Math. 20 (1967), 493-519.

[LS1] V. K. Le and K. Schmitt, Minimization problems for noncoercive functionals subject to constraints, Trans. A. M. S. 347 (1995), 4485-4513.

[LS2] V. K. Le and K. Schmitt, Minimization problems for noncoercive functionals subject to constraints II, Advances in Diff. Eq., to appear.

[MW] J. Mawhin and M. Willem, Critical Point Theory and Hamiltonian Systems, Springer, New York, 1989.

[PS] D. Pascali and J. Sburlan, Nonlinear Mappings of Monotone Type, Sijthoff and Noordhoff, Bucharest, 1978.

[PM] M. A. delPino and R. F. Manasevich, Global bifurcation from the eigenvalues of the p-Laplacian, J. Diff. Equations 92 (1991), 226-251.

[R] T. R. Rockafellar, Convex Analysis, Princeton University Press, New Jersey, 1970.

[S] M. Schatzman, Problèmes aux limites non linéaires semi-coercifs, C. R. Acad. Sc. Paris 275 (1972), 1305-1308.

[St] M. Struwe, Variational Methods and their Applications in Nonlinear Partial Differential Equations, Springer, New York, 1990.

[T] S. L. Troyanski, On locally uniformly convex and differentiable norms in certain non-separable Banach spaces, Studia Math. 37 (1971), 173-180. 